\title{
Une arme à double tranchant : le déploiement du portrait de la reine dans les édifices gouvernementaux et dans les caricatures québécoises
}

\author{
Josée Desforges
}

Volume 40, numéro 1, 2015

URI : https://id.erudit.org/iderudit/1032747ar

DOI : https://doi.org/10.7202/1032747ar

Aller au sommaire du numéro

\section{Éditeur(s)}

UAAC-AAUC (University Art Association of Canada | Association d'art des universités du Canada)

\section{ISSN}

0315-9906 (imprimé)

1918-4778 (numérique)

Découvrir la revue

\section{Citer cet article}

Desforges, J. (2015). Une arme à double tranchant : le déploiement du portrait de la reine dans les édifices gouvernementaux et dans les caricatures québécoises. RACAR : Revue d'art canadienne / Canadian Art Review, 40(1), 1-16. https://doi.org/10.7202/1032747ar
Résumé de l'article

Since the recent increase in the number of representations of Queen Elizabeth II in Canadian government buildings, Quebec cartoonists have seized the motif of the royal portrait to denounce the Harper government's uses of the sovereign's image. Drawing on Louis Marin's definition of representation, this article considers the powers granted by the state to the Queen's portrait and highlights its critique in Quebec caricatures. Indeed, while the portrait of the Queen allows the Prime Minister to legitimize his authority, its satirical representations denounce the government's re-actualization of an absent monarchy. The royal portrait is then satirized both as a powerless object and as a weapon threatening to overshadow other Canadian political and religious symbols.
Tous droits réservés (C) UAAC-AAUC (University Art Association of Canada | Association d'art des universités du Canada), 2015
Ce document est protégé par la loi sur le droit d'auteur. L'utilisation des services d’Érudit (y compris la reproduction) est assujettie à sa politique d'utilisation que vous pouvez consulter en ligne.

https://apropos.erudit.org/fr/usagers/politique-dutilisation/ 


\title{
Une arme à double tranchant : le déploiement du portrait de la reine dans les édifices gouvernementaux et dans les caricatures québécoises
}

\author{
Josée Desforges, Université du Québec à Montréal
}

\begin{abstract}
Since the recent increase in the number of representations of Queen Elizabeth II in Canadian government buildings, Quebec cartoonists have seized the motif of the royal portrait to denounce the Harper government's uses of the sovereign's image. Drawing on Louis Marin's definition of representation, this article considers the powers granted by the state to the Queen's portrait and highlights its critique in Quebec caricatures. Indeed, while the portrait of the Queen allows the Prime Minister to legitimize his authority, its satirical representations denounce the government's re-actualization of an absent monarchy. The royal portrait is then satirized both as a powerless object and as a weapon threatening to overshadow other Canadian political and religious symbols.
\end{abstract}

$\mathrm{L}$ 26 juin 2011, deux œuvres du peintre québécois Alfred Pellan (1906-1988) qui se trouvaient dans le hall d'entrée du ministère des Affaires étrangères, Commerce et Développement Canada (MAECD) sont remplacées par une imposante photographie de la reine Élisabeth II (figs. 1-3). Lorsque Canada Est (1942) et Canada Ouest (1942-1943) sont décrochées du mur gris devant lequel est situé un poste de sécurité qui fait office d'accueil, des traces de décoloration apparaissent, marquant l'endroit où les deux œuvres étaient exposées depuis près de quarante ans. La substitution avait été organisée par le Cabinet du premier ministre Stephen Harper récemment élu à la tête d'un gouvernement majoritaire aux élections du 2 mai 2011 dont faisait alors partie le ministre des Affaires étrangères, John Baird ${ }^{1}$. Peu de temps après cet évènement qui signale une perturbation iconographique, le gouvernement ordonne à ses ambassades d'afficher un portrait de la souveraine ${ }^{2}$. Le remplacement des œuvres de Pellan donne ainsi le coup d'envoi à une multiplication du portrait royal dans les édifices gouvernementaux canadiens.

Les caricaturistes québécois se sont rapidement emparés de cette suite d'évènements pour parodier l'intérêt marqué du gouvernement conservateur envers la monarchie britannique. Depuis le 26 juillet 2011, date à laquelle l'évènement est révélé publiquement dans un article de Jennifer Ditchburn ${ }^{3}$, soixantetrois caricatures québécoises représentant le personnage du premier ministre Stephen Harper accompagné du portrait royal ont été répertoriées ${ }^{4}$. Il n'est pas surprenant que les Québécois se montrent si sensibles à l'intérêt que porte le gouvernement Harper aux symboles de la monarchie, puisque le régime britannique est synonyme de conquête dans l'histoire canadiennefrançaise. C'est, du moins, ce que rappellent avec force certains politiciens d'allégeance séparatiste qui s'approprient l'affaire des Pellan à des fins politiques : Louis Plamondon du Bloc québécois, Thomas Deshaies du Mouvement progressiste pour l'indépendance du Québec et Yves-François Blanchet porte-parole péquiste en matière de culture5.

Les soixante-trois caricatures québécoises se sont avérées suffisantes pour donner une autonomie au motif du portrait de la reine. En effet, ce dernier s'est rapidement dégagé de l'affaire des Pellan qui l'a fait naitre à titre de motif récurrent dans les satires graphiques. Dès les premières semaines qui suivent la substitution des Pellan, les caricaturistes extirpent le portrait de la reine hors des murs de l'édifice Pearson; ils transforment alors l'image royale en l'attribut politique privilégié de Stephen Harper. Suivant le premier ministre dans ses déplacements, de son bureau à sa chambre à coucher, en passant par un voyage en Afrique et une visite dans le Grand Nord canadien, le portrait devient alors une nouvelle poire philiponnienne, traçant son propre parcours iconographique, avec sa genèse et son déploiement dans l'intercaricaturalité québécoise, c'est-à-dire dans la production de plusieurs caricaturistes québécois, peu importe leur journal d'attache ou leur inclinaison idéologique ${ }^{6}$. Lautoréférentialité du motif du portrait ne signifie toutefois pas qu'il y a une coupure pure ${ }^{7}$ avec le remplacement des Pellan par un portrait de la reine. Dans les caricatures québécoises, le portrait demeure associé, de près ou de loin, à son contexte d'émergence, notamment lorsque les caricaturistes le présentent comme un substitut non pas des œuvres de Pellan, mais des pouvoirs monarchiques que souhaite s'approprier le personnage du premier ministre. À travers le rappel de l'action de substituer, les caricatures mettent ainsi en exergue les pouvoirs que les Conservateurs accordent aux images quand ils remplacent les œuvres accrochées dans les édifices gouvernementaux, et ce, en dépit des coupes budgétaires effectuées à l'endroit de la culture et des arts en particulier ${ }^{8}$.

Tout en soulevant la question des différents pouvoirs attribués par le gouvernement conservateur aux représentations photographiques et picturales de la reine, ce texte analysera de quelles manières les caricatures québécoises critiquent ces utilisations de l'image tout en participant à la dissémination du portrait royal ${ }^{9}$. Autrement dit, il tentera de répondre à la question suivante : comment les caricaturistes reprennent-ils pour leur propre dessein le portrait de la reine et les pouvoirs qui lui sont attribués dans certains établissements gouvernementaux?

Afin de répondre à cette question, les différents pouvoirs attribués au portrait royal dans les établissements 


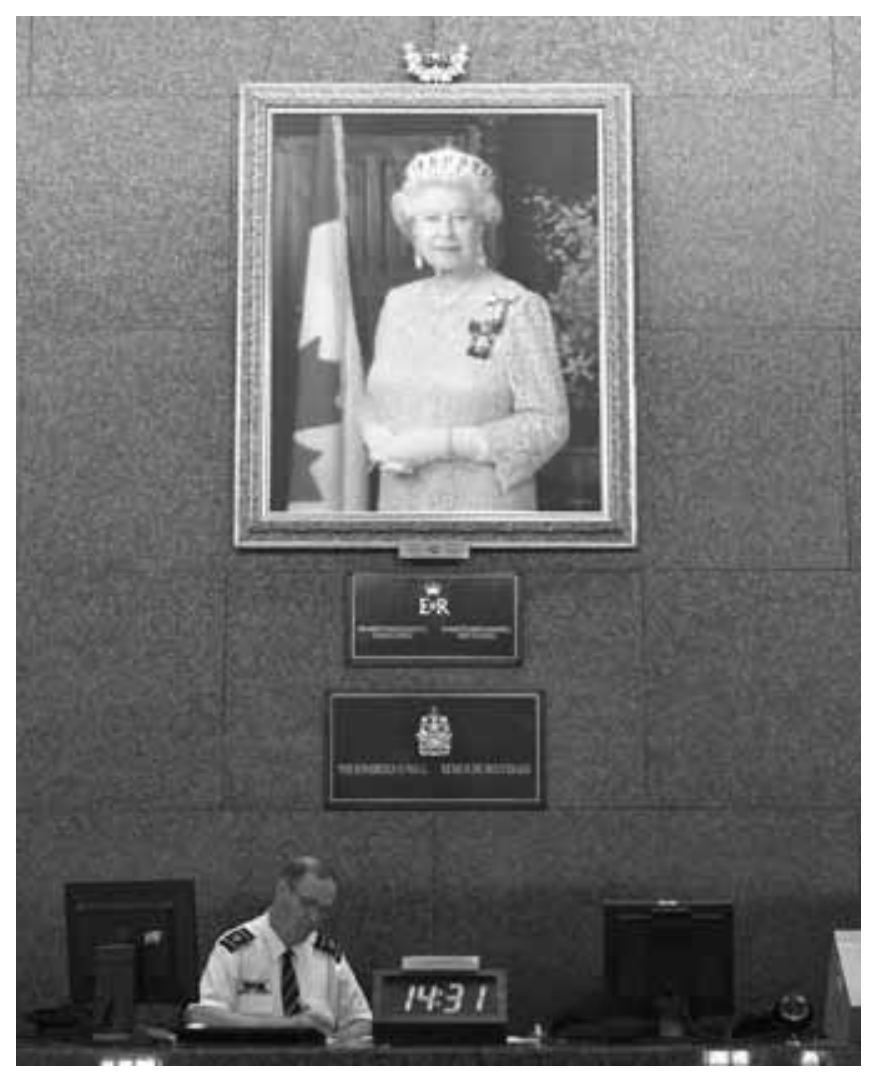

Figure I. Michael Bedford, Photographie de la reine Élisabeth II lors de son jubilé d'or, octobre 2002, photographie marouflée sur toile, $182 \times 145 \mathrm{~cm}$, Ministère des Affaires étrangères, Commerce et Développement Canada (Photo : La Presse Canadienne / Adrian Wyld).

gouvernementaux seront analysés en prenant comme point de départ les théories de Louis Marin sur le triple pouvoir la représentation. Ces pouvoirs - la substitution, la légitimation et la force économisée - seront considérés pour étudier la photographie de la reine qui a remplacé les deux œuvres d'Alfred Pellan, de même qu'un portrait de la souveraine, peint à l'acrylique par l'artiste ontarien Phil Richards, présentement exposé dans la salle de bal de Rideau Hall, lieu où sont assermentés les premiers ministres canadiens et leurs Cabinets. La discussion sur les trois pouvoirs de la représentation permettra d'introduire deux méthodes paradoxales qui sont privilégiées par les caricaturistes qui constituent l'intercaricaturalité québécoise lorsqu'il est question de satiriser l'intérêt du gouvernement conservateur à l'égard des images royales. L'une consiste à ridiculiser le premier ministre en présentant le portrait royal comme un objet vide de pouvoir - un fétiche inanimé ou un grigri dénué de magie, mais utilisé malgré tout pour régler des conflits religieux, sociaux et environnementaux. L'autre prend forme dans la mise en scène du premier ministre qui réussit à animer le portrait de la reine et à en faire un emblème canadien susceptible de remplacer d'autres symboles en place. Dans ce deuxième cas, la satire adopte un ton prophétique et la multiplication du portrait devient anxiogène. Quelles seront les prochaines substitutions iconographiques? À quels autres symboles canadiens s'attaqueront le premier ministre et ses portraits de la reine? Ce texte est donc structuré par une double alternance. D'abord, un va-et-vient entre l'utilisation du portrait de la reine par l'État dans les édifices gouvernementaux et l'utilisation du portrait de la reine comme attribut du personnage de Stephen Harper dans les caricatures québécoises. Ensuite, un balancement entre la présentation au sein des caricatures québécoises du portrait royal d'abord comme un objet vide de pouvoir, puis comme un objet de pouvoir, ceci afin de ridiculiser l'opération politique des Conservateurs tout en exposant une angoisse collective.

\section{L'évènement initiateur}

Les gouaches sur toile montées sur panneaux qui ornaient le mur d'accueil du MAECD n'ont été connues de la majorité des Canadiens et des Canadiennes qu'à la suite de leur disparition. $\mathrm{Si}$ le lieu où elles se trouvaient est accessible à tous, le hall d'entrée du MAECD n'est pourtant habituellement visité que par les employés du gouvernement, les ambassadeurs étrangers et les invités de marque, comme la famille royale. Le public à la recherche d'art et d'histoire qui fréquente les édifices gouvernementaux privilégie plutôt les résidences du gouverneur général du Canada - Rideau Hall et la Citadelle à Québec -, des lieux visités par 114000 personnes chaque année ${ }^{10}$. Même John Porter, l'ancien directeur du Musée national des beaux-arts du Québec (MNBAQ) où est hébergée une importante collection d'œuvres d'Alfred Pellan, admet ne pas avoir connu l'existence de Canada Est et Canada Ouest et encore moins leur histoire avant leur retrait du MAECD en $2011^{11}$.

Les œuvres Canada Est et Canada Ouest ont été commandées par l'ambassadeur canadien Jean Désy (1893-1960) en 1941 pour représenter le Canada dans sa légation à Rio de Janeiro, au Brésil12. Elles sont ensuite rapatriées au Canada après le déménagement de l'ambassade canadienne à Brasilia en avril 1972, qui n'avait plus l'espace suffisant pour les loger. Les deux tableaux sont alors accrochés dans l'édifice Pearson qui abrite le MAECD, rue Sussex, à Ottawa, face à l'entrée principale du bâtiment, endroit où elles continuent à être associées aux politiques internationales canadiennes.

Les deux toiles schématisent les paysages de l'Ouest et de l'Est canadien. Les couleurs vives uniformisent les divers éléments figuratifs aux perspectives plurielles qui parsèment les deux compositions. Lemploi de couleurs vibrantes et de perspectives fragmentées sont caractéristiques du style 


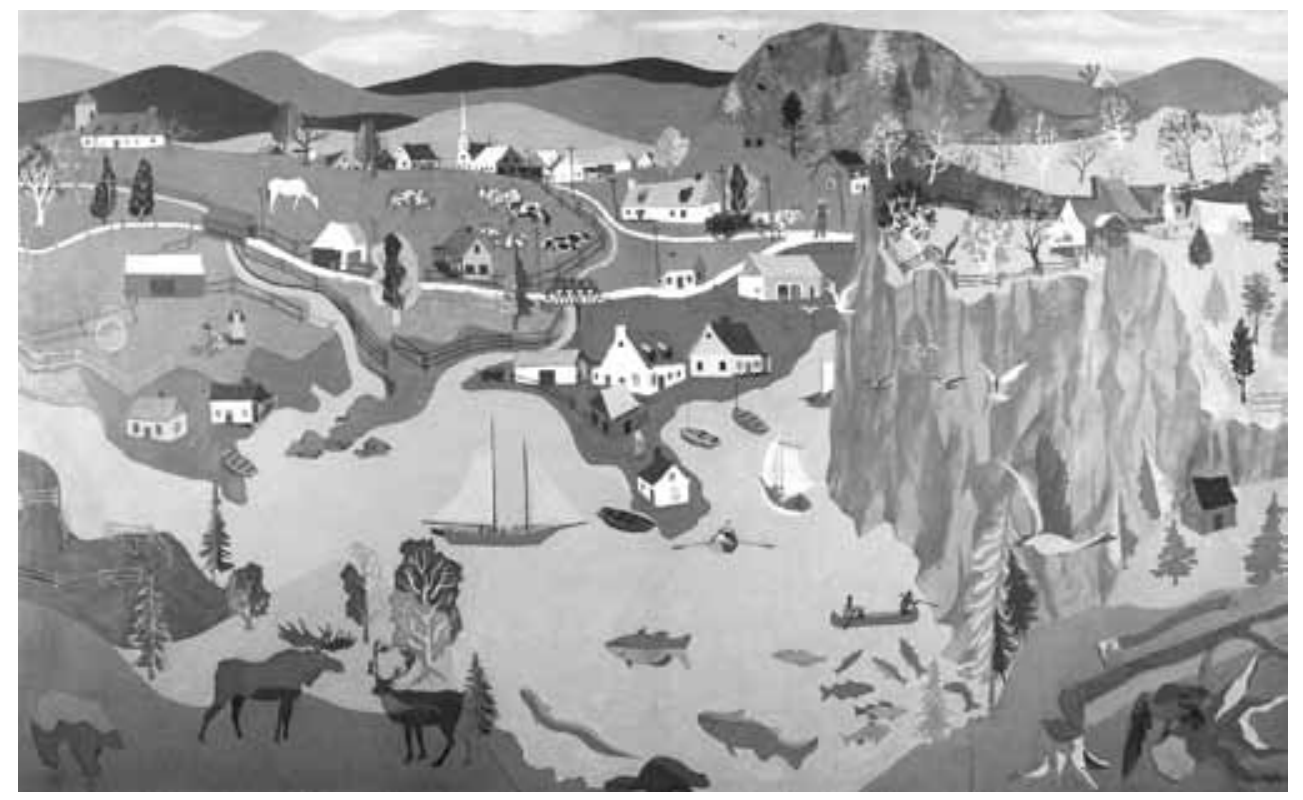

Figure 2. Alfred Pellan, Sans titre (Canada Est), 1942, gouache sur toile montée sur panneau de bois, 194,3 × 307,3 cm, ministère des Affaires étrangères, Commerce et Développement Canada. @ Succession Alfred Pellan / Sodrac (2015).

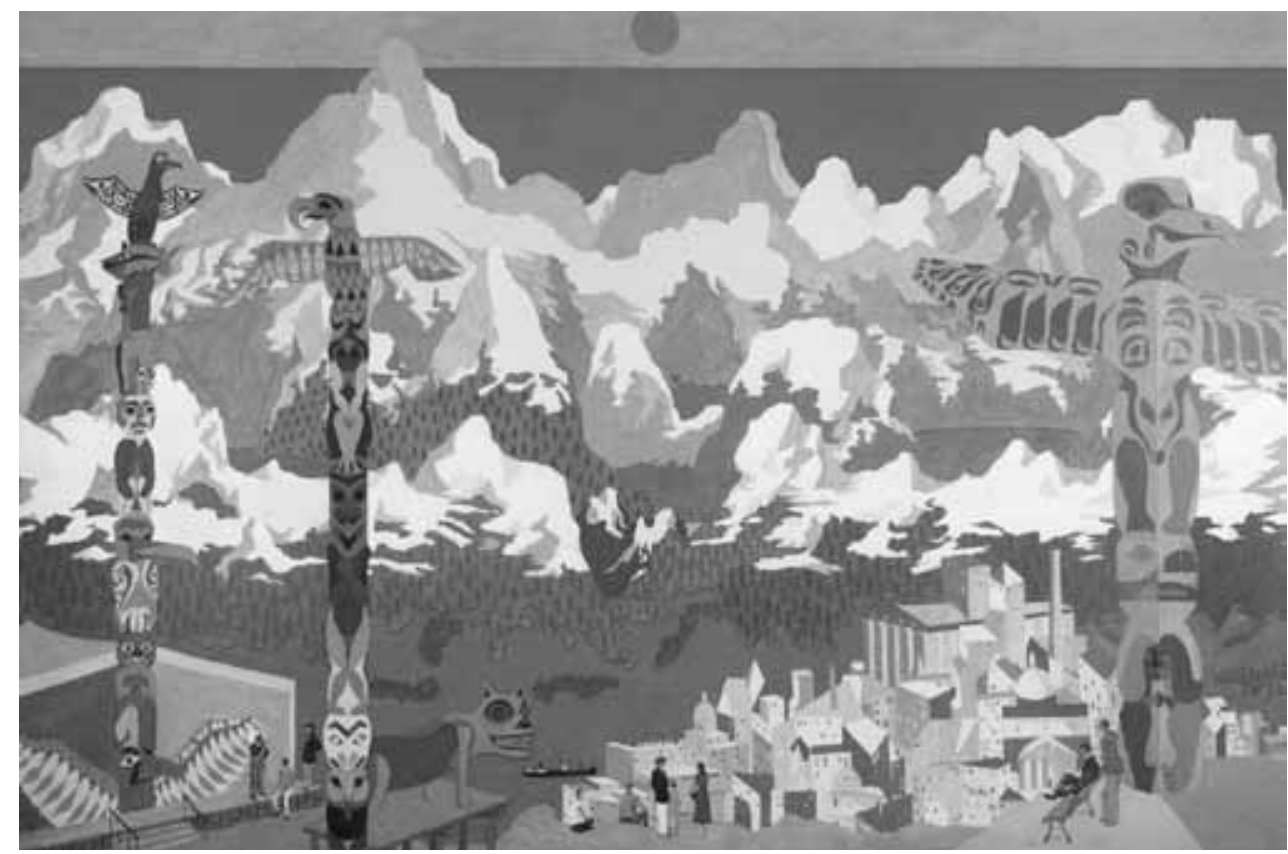

Figure 3. Alfred Pellan, Sans titre (Canada Ouest), 1942-1943, gouache sur toile montée sur panneau de bois, $193 \times 304,8$ cm, ministère des Affaires étrangères, Commerce et Développement Canada. @ Succession Alfred Pellan / Sodrac (2015). 
moderne éclectique que développe Pellan lors de son long séjour à Paris entre 1926 et 1940 , et qui prend subitement fin avec le début de la Seconde Guerre mondiale. La thématique canadienne du diptyque, quant à elle, n'est pas courante dans la production de l'artiste et met en évidence le statut de commande des œuvres ${ }^{13}$. Le choix d'Alfred Pellan en guise de représentant du Canada dans le contexte de missions diplomatiques internationales n'a rien d'étonnant. À son retour de Paris, le peintre est considéré par plusieurs critiques canadiens comme un artiste de renommée internationale qui s'inscrit dans la lignée de Picasso ${ }^{14}$.

La soudaine notoriété acquise par Canada Ouest et Canada Est suite à leur remplacement conduit le MNBAQ à les exposer du 20 février au 16 juin 2014, dans le cadre de l'inauguration de l'exposition Alfred Pellan, Le rêveur éveillé. Lorsque le prêt au musée arrive à échéance, le gouvernement décide de réinstaller les œuvres dans le hall d'entrée du MAECD, mais sur un mur adjacent à celui occupé par le portrait de la reine qui demeure toujours en place ${ }^{15}$. Il pouvait difficilement en être autrement, car l'endroit où se trouve maintenant la photographie royale a été baptisé en 2011 " the sovereign's wall ", le mur de la souveraine, comme l'indique l'une des plaques qui se trouvent sous le portrait officiel. Sa nouvelle vocation - rendre hommage à " notre digne chef d'État, la reine Elizabeth II 16 " - contribue à hiérarchiser les différents murs du hall d'entrée : celui derrière le bureau d'accueil devient un dispositif officiel pour représenter la monarchie au Canada.

La photographie du buste d'Élisabeth II mesure environ $182 \times 145 \mathrm{~cm}$. Elle fait donc de la reine une souveraine bien plus grande que nature ${ }^{17}$. L'image a été saisie par Michael Bedford lors de la visite d'Élisabeth II au Canada en octobre 2002, à l'occasion de son jubilé d'or ${ }^{18}$. La photographie, retrouvée dans le sous-sol du Parlement en 2006, a alors été dépoussiérée et accrochée dans la salle du Conseil des ministres ${ }^{19}$. Le portrait a ensuite remplacé les deux œuvres de Pellan le 30 juin 2011 afin d'accueillir le duc et la duchesse de Cambridge (le prince William et Kate Middleton) lors de leur visite au Canada. Le départ du couple royal n'a pourtant pas provoqué un retour à l'ancienne décoration du hall d'entrée, même si la photographie a dû être retournée au Parlement à la suite d'une demande de la part du Cabinet du premier ministre ${ }^{20}$. En effet, le MAECD semble avoir été contraint de se procurer une « copie » de la photographie et de s'assurer que le portrait appartenant au Cabinet revienne à bon port, peu importe les coûts engendrés par son déplacement ${ }^{21}$. Il semble qu'une aura nimbe cette photographie de Bedford pour qu'elle doive se trouver près du corps du premier ministre, au centre de l'édifice du Parlement ${ }^{22}$. Certains caricaturistes reprendront ce rapport de proximité entre le portrait de la reine et le premier ministre en le pimentant d'un discours à teneur sexuelle.
Une seconde substitution d'œuvres d'art impliquant une représentation de la reine Élisabeth II est survenue après l'affaire des Pellan. Le remplacement s'est produit en juin 2012 sur le mur nord de la salle de bal de la résidence du gouverneur général, Rideau Hall. Androgyny (1983) de l'artiste amérindien Norval Morriseau (1931-2007) qui occupait l'endroit depuis 2008 a été remplacée par un portrait de la reine mesurant $315 \times 224 \mathrm{~cm}^{23}$. Intitulé Le Portrait du jubilé de diamant de Sa Majesté la reine Élisabeth II, le tableau peint en 2012 par l'artiste ontarien Phil Richards (1951-) se trouve à un endroit stratégique. Non seulement la salle de bal est-elle le lieu où sont assermentés le premier ministre et ses ministres par le gouverneur général après chaque élection et chaque remaniement ministériel, mais l'acte performatif du serment se produit face au mur nord. Dorénavant, les élus qui entrent en fonction le font devant un portrait colossal de la reine Élisabeth II24.

Ce regain d'intérêt pour la monarchie est un changement de cap de la part du gouvernement canadien. En effet, les liens entre le Canada et l'Angleterre ont été relâchés à plusieurs reprises au cours du XXe siècle, notamment après la Seconde Guerre mondiale sous l'influence de gouvernements majoritairement libéraux. Plusieurs évènements soulignent l'autonomisation grandissante du Canada vis-à-vis de l'Angleterre : nommons ici la présence du Canada au sein de l'Organisation du traité de l'Atlantique Nord; la participation active du Canada à la création de l'Organisation des Nations Unies; l'adoption de l'unifolié en 1965; l'abandon des désignations Aviation royale du Canada et Marine royale canadienne en 1968; le rapatriement la Constitution canadienne en 1982; l'accord du Lac Meech en 199025. En restituant le terme « royal » pour certaines instances canadiennes ${ }^{26}$, de même qu'en multipliant le portrait de la reine dans les édifices gouvernementaux tant à l'intérieur qu'à l'extérieur du pays, le gouvernement de Stephen Harper retisse les liens symboliques entre le Canada et la monarchie britannique. Il déplace ainsi certaines valeurs mises en place par les Libéraux depuis Lester B. Pearson (tel que la notion de multiculturalisme et l'image du Canada comme gardien de la paix). Ce retour à un passé prépearsonien permet au gouvernement conservateur de repositionner le Canada dans l'empire anglo-saxon (l'anglosphere) tout en promouvant un système politique autoritaire27.

L'intérêt particulier porté au portrait de la reine par le gouvernement conservateur s'inscrit également dans le schème développé par Louis Marin qui accorde à la représentation le pouvoir de présenter quelque chose "à la place " d'une autre et aussi de légitimer le sujet qu'elle représente. De plus, le portrait d'un souverain ou d'une souveraine est une représentation de pouvoir, c'est-à-dire, d'une force économisée. En effet, la représentation d'un pouvoir politique montre que ce dernier détient une force (monétaire, militaire, législative) 
sans que celle-ci soit utilisée. Cette force contenue et exposée à travers des signes engendre un climat de peur et de respect envers l'autorité28.

\section{Substituer la reine par le pouvoir de sa représentation}

Le premier pouvoir évoqué par Marin, qui réside dans la présentation d'une chose "à la place " d'une autre, permet de "faire comme si " un absent était présent à travers sa représentation. Louis Marin n'est pas le seul théoricien à avoir souligné la capacité du portrait à rendre présent ce qui est absent, comme l'ont récemment rappelé Christine Tauber et Antonio Pinelli. Pline l'Ancien y faisait déjà allusion dans l'histoire de la fille du potier Butadès de Sicyone qui conservait l'image de son amoureux lorsqu'il partait au loin ${ }^{29}$. Peter Burke fait également référence au pouvoir de suppléance de la représentation lorsqu'il annonce que tourner le dos à un portrait du roi Louis XIV revenait à tourner le dos au roi ${ }^{30}$.

Les portraits de la reine en sol canadien s'inscrivent en partie dans les traditions du portrait royal en Angleterre et en France à la fin du Moyen-Âge et sous Louis XIV. S'il est possible de tourner le dos aux portraits de la souveraine sans crainte de commettre un crime de lèse-majesté, les représentations de la reine sont néanmoins instituées d'un pouvoir de remplacement. Ils agissent à la place de la reine, notamment lorsque les ministres prêtent serment devant le portrait peint par Phil Richards à Rideau Hall. Deux substituts de la reine participent donc à l'assermentation : le gouverneur général qui parle au nom de la souveraine, et le portrait de la reine qui regarde la scène, en tant que témoin.

Les caricaturistes n'ont pas eu à attendre l'arrivée du portrait de Phil Richards pour s'attaquer au rôle de substitut du portrait de la reine. En prenant bien soin d'isoler le portrait royal dans un cadre afin d'insister sur son caractère d'objet, ils le mettent en scène accompagnant Stephen Harper, qui adopte envers lui un comportement fétichiste dysfonctionnel. En cherchant à animer un objet inanimé, le personnage du premier ministre se révèle comme n'arrivant pas à faire la distinction entre la reine et sa représentation.

Dans les caricatures, le lieu occupé par le portrait intensifie l'intimité que souhaite nouer le personnage du premier ministre avec la souveraine. Dans le cabinet de travail d'Harper, le portrait royal remplace les photographies de famille qui trônent sur le bureau et les diplômes qui tapissent les murs ${ }^{31}$. Le portrait de la reine est également exposé dans les appartements privés du premier ministre, voire dans sa chambre à coucher, au-dessus du lit conjugal ${ }^{32}$. Le caricaturiste Garnotte pousse l'analogie du couple plus loin en évoquant les fantasmes sexuels qu'entretient le personnage du premier ministre avec la reine d'Angleterre. Dans une caricature intitulée Jubilé de diamant : Postes Canada

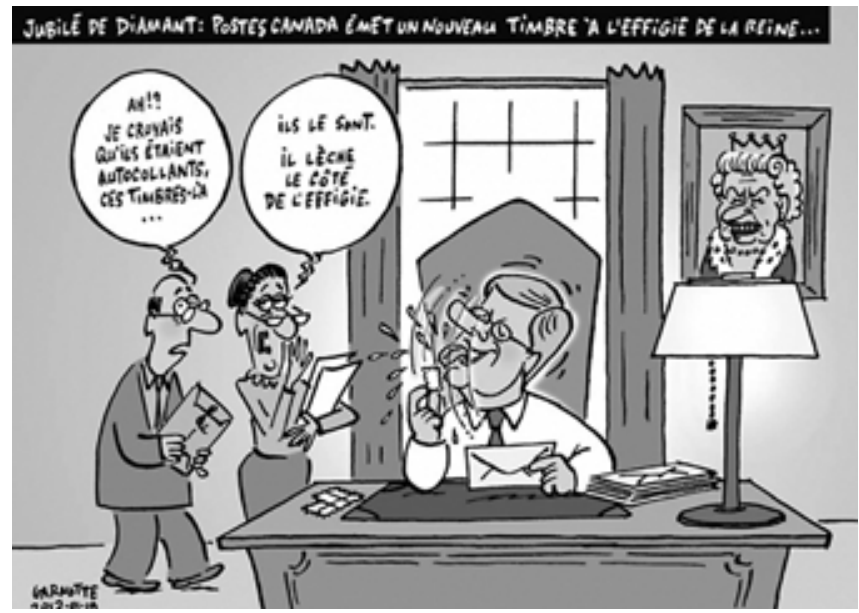

Figure 4. Garnotte (Michel Garneau), Jubilé de diamant: Postes Canada émet un nouveau timbre à l'effigie de la reine, Le Devoir, 18 janvier 2012, avec l'amabilité de l'artiste.

émet un nouveau timbre à l'effigie de la reine, Harper est représenté assis à son bureau, léchant un timbre avec vigueur, comme le révèlent les traits entourant la tête du politicien qui simulent le mouvement (fig. 4). Le portrait de la souveraine, accroché sur un mur derrière la scène, exhibe au spectateur la face cachée de l'objet sexuellement investi, car il s'agit bien ici d'un désir charnel qu'éprouve le protagoniste envers la représentation royale. Les hochements de tête frénétiques du premier ministre, ses yeux fermés, son sourire et les gouttes de salive qui s'échappent de sa bouche témoignent du plaisir physique éprouvé par le premier ministre. Le fantasme sexuel - qui consiste à lécher la reine - est transféré dans un objet, un fétiche. Les théories de Freud sur le fétichisme comme aberration sexuelle sont encore d'actualité : dans cette caricature de Garnotte, le fétiche dénonce les comportements déphasés du premier ministre envers une monarchie réactualisée par l'image 33 .

L'union entre le personnage de Stephen Harper et le portrait de reine Élisabeth II semble déjà avoir été consommée dans une caricature d'Aislin qui précède pourtant le remplacement des œuvres de Pellan par une photographie de la reine (fig. 5). Le premier ministre y pose en avant-plan, exhibant des symboles royaux : un manteau de couronnement, un sceptre orné d'une feuille d'érable et une couronne ${ }^{34}$. Les proportions de cette dernière contrastent avec la tête du premier ministre qui est, à son tour, trop volumineuse pour son corps. Or, le corps du premier ministre est plus imposant que celui de la reine, en arrière-plan, qui conserve son statut d'objet, soit celui de portrait encadré. La souveraine ne porte pas de couronne, elle ne tient aucun sceptre et elle est vêtue non pas d'un manteau 


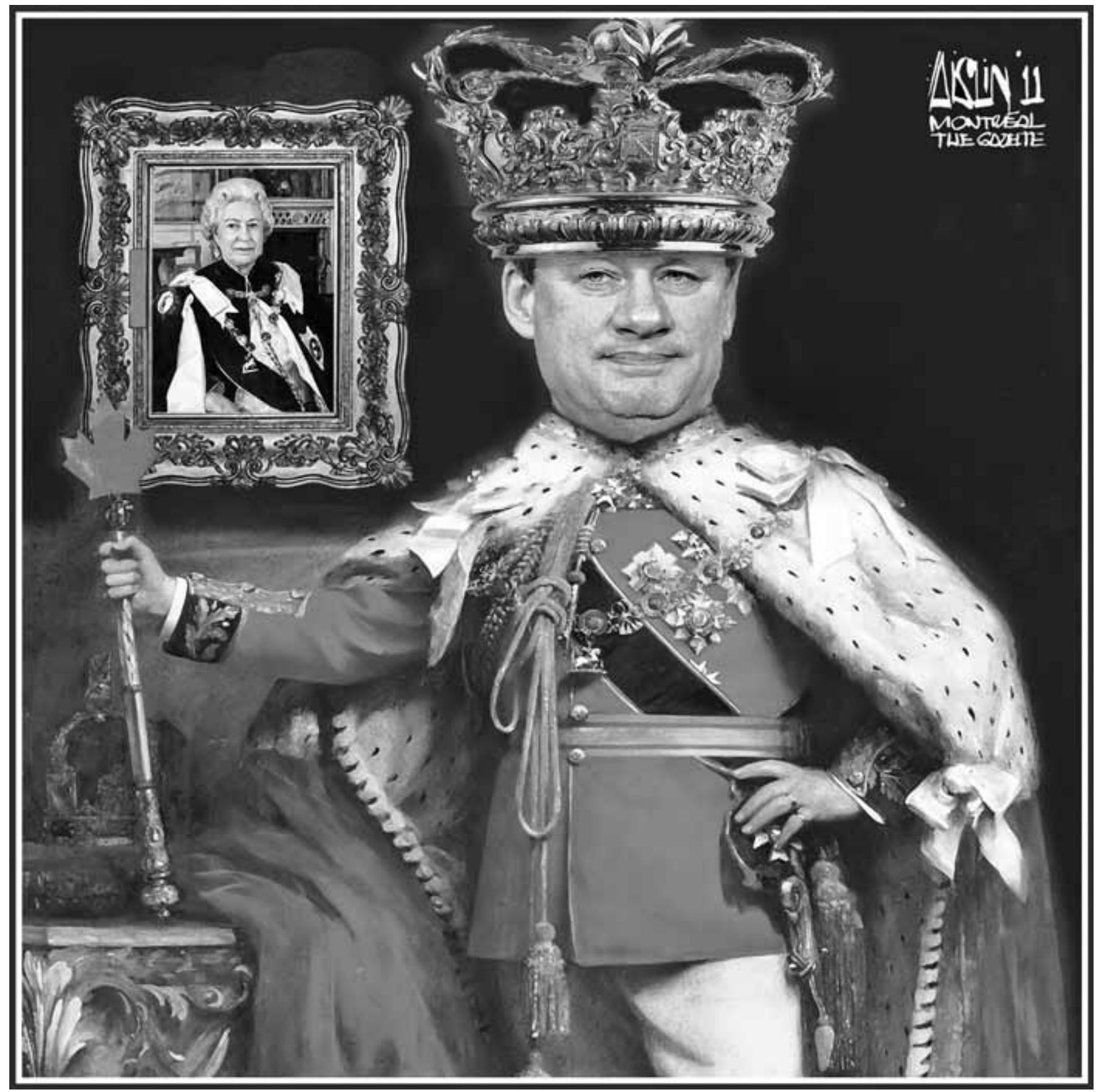

Figure 5. Aislin (Terry Mosher), sans titre, The Gazette, 5 avril 20II, avec l'amabilité de l'artiste. 
de couronnement, mais de l'habit de l'Ordre de la jarretière. Ces jeux de taille et d'attributs laissent supposer que Stephen Harper occupe une position supérieure à celle de la reine. Si le premier ministre est le roi, Élisabeth II serait-elle son épouse? L'habit de l'Ordre de la jarretière porté par Élisabeth II pourrait ainsi évoquer un certain lien sexuel entre les deux protagonistes puisqu'une des légendes qui entourent la conception de l'Ordre au XIV ${ }^{e}$ siècle est elle-même teintée de sexualité. En dansant avec le roi Édouard III lors d'un bal à Calais, la comtesse de Salisbury perdit sa jarretière. Après l'avoir ramassée, le roi la réinstalla à la jambe de la comtesse, répondant aux murmures des hôtes en disant " Honi soit qui mal y pense ». La célèbre phrase est devenue la devise de l'ordre prestigieux. Dans cette image, le personnage du premier ministre Harper est également associé à un membre de la famille royale. En fait, la caricature d'Aislin est une citation du portrait d'Édouard VII, le fils de la reine Victoria, peint par Samuel Luke Fildes (1843-1927) en 190235. Il y a effectivement un air de famille entre Harper et Élisabeth II, à la fois dans leurs regards et dans leurs bouches crispées. Néanmoins, la volonté d'Harper de s'approcher de la reine en devenant lui-même un portrait et en reprenant les mimiques de la reine ne mène pas à une humanisation de cette dernière ${ }^{36}$. Elle reste immobile, sans montrer le moindre signe de vie, malgré les efforts du premier ministre pour l'animer. Les caricaturistes désamorcent ainsi l'un des pouvoirs de la représentation royale. Le portrait de la reine ne sera jamais la reine, si ressemblant soit-il.

\section{Légitimer le pouvoir par le pouvoir de la représentation}

En étant témoin du serment du premier ministre et de son Cabinet ainsi qu'en accueillant les invités de marque dans le hall d'entrée du MAECD et dans les ambassades canadiennes, les représentations de la souveraine dans les établissements gouvernementaux acquièrent un pouvoir d'attestation. C'est ici qu'entre en jeu le deuxième pouvoir de la représentation défini par Marin. Celui-ci consiste à re-présenter quelque chose, à intensifier une présence par la duplication d'un dispositif de représentation, ce qui mène à une légitimation de la représentation et de son sujet ${ }^{37}$. Les portraits de la reine relégitiment sans cesse l'autorité du premier ministre. L'incontestabilité de cette autorité - car nommée par la reine - est surveillée par le regard de la souveraine qui fait, en quelque sorte, office de justice. La fusion entre le corps de la souveraine et celui de la justice s'inscrit dans une longue tradition analysée par Ernst Kantorowicz dans son étude sur les deux corps (naturel et politique) du roi à la fin du moyen-âge ${ }^{38}$. Cependant, dans le contexte du Canada au $\mathrm{XXI}^{\mathrm{e}}$ siècle, un nouvel acteur entre en jeu. Le pouvoir législatif de la représentation royale ne sert pas qu’à légitimer le pouvoir de la reine, mais aussi celui du premier ministre. Ce dernier

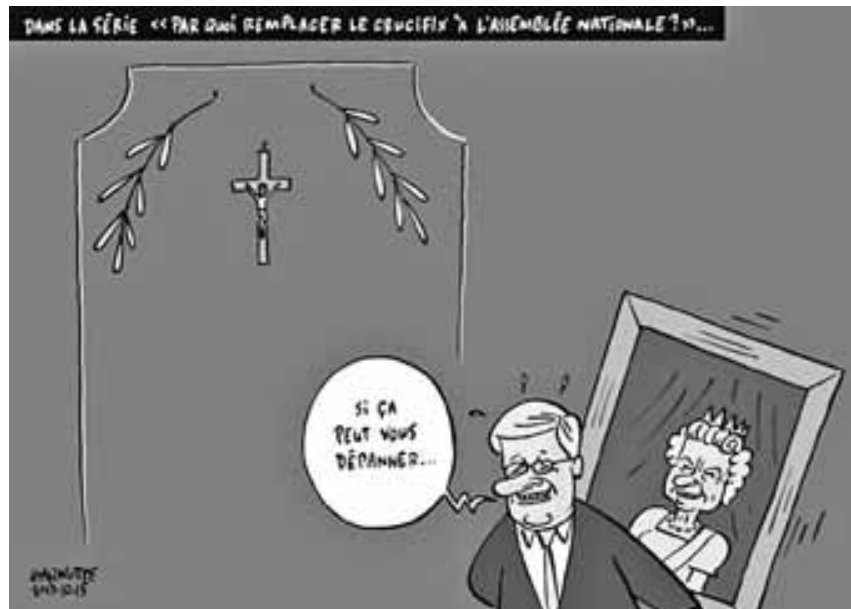

Figure 6. Garnotte (Michel Garneau), Dans la série « Par quoi remplacer le crucifix à l'Assemblée nationale? », Le Devoir, 15 octobre 2013, avec l'amabilité de l'artiste.

aura ensuite les mains libres pour légiférer, compte tenu du rôle davantage symbolique joué par la monarchie au Canada ${ }^{39}$. Le portrait de la reine tient donc lieu du pouvoir législatif que la souveraine ne détient que de façon nominale dans les prises de décisions quotidiennes à la Chambre des communes.

L'échec du pouvoir législatif du portrait royal est une thématique récurrente dans l'intercaricaturalité québécoise. Le portrait est souvent présenté comme un objet caduc lorsque manipulé par le premier ministre qui le brandit telle une amulette dans l'espoir de régler toutes sortes de conflits politiques, religieux et environnementaux. Ces mises en situation façonnent le personnage du premier ministre en inculte qui ne se rend guère compte de l'inefficacité de son grigri.

L'un des problèmes que tente, sans succès, de résoudre Stephen Harper avec l'image royale est le sort réservé au crucifix de l'Assemblée nationale du Québec. Dans la caricature de Garnotte intitulée Dans la série "Par quoi remplacer le crucifix à l'Assemblée nationale?" "le premier ministre fédéral s'ingère dans la Charte des valeurs, un projet de loi provinciale controversé mis sur pied par le Parti québécois en 2013 (fig. 6). La Charte propose le rejet du port d'objets religieux jugés ostentatoires par les membres du personnel des organisations publiques, afin qu'ils puissent " faire preuve de neutralité » dans l'exercice de leurs fonctions ${ }^{40}$. Dans le même ordre d'idées, les opposants, mais aussi certains partisans du projet de loi, ont demandé la suppression du crucifix fixé au-dessus de la chaise du président de l'Assemblée nationale. Le refus du Parti québécois face à cette demande est fondé sur le présupposé statut patrimonial du crucifix. Dans la caricature de Garnotte, Harper tente de 


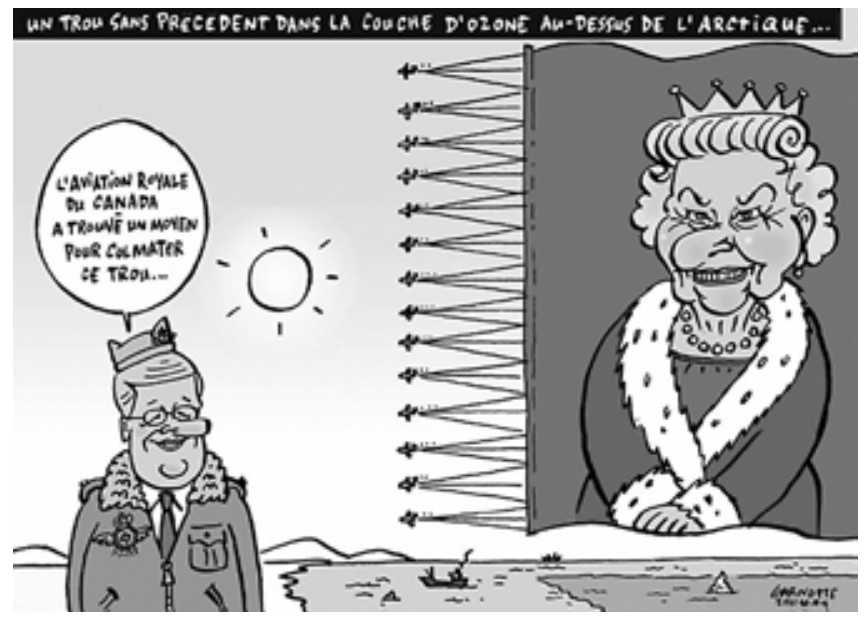

Figure 7. Garnotte (Michel Garneau), Un trou sans précédent dans la couche d'ozone au-dessus de l'Arctique, Le Devoir, 4 octobre 20II, avec l'amabilité de l'artiste.

remplacer le crucifix par un portrait de la reine, qui n'est pas dans le répertoire du Patrimoine culturel du Québec. Aussi, en plus de remplacer un crucifix de dimension relativement moindre par un symbole bien plus ostentatoire, la proposition d'Harper ne règlerait en rien le litige. En effet, en tant que représentante de Dieu sur la terre, la reine constitue elle aussi un symbole religieux.

Dans une autre caricature de Garnotte datée du 4 octobre 2011 et intitulée Un trou sans précédent dans la couche d'ozone au-dessus de l'Arctique, le premier ministre en mission dans le Nord canadien explique fièrement la solution originale proposée par l'armée de l'air à l'appauvrissement de la couche d'ozone : "Laviation royale du Canada a trouvé un moyen pour colmater ce trou " (fig. 7). Telle une immense bâche, l'image de la reine tirée par une quinzaine d'avions s'apprête à obturer un orifice gigantesque. Or, la forme ronde qui brille au centre-gauche de l'image n'est pas le trou de la couche d'ozone. Il s'agit du soleil. La méprise de l'armée de l'air canadienne ralentira peut-être le réchauffement climatique, mais elle assombrira aussi la planète. Le personnage d'Harper ne s'aperçoit de rien et croit plutôt faire d'une pierre deux coups : d'une part, régler des problèmes climatiques, et d'autre part, assurer la possession de l'Arctique, un espace convoité par le gouvernement conservateur, en implantant le portrait royal qui fait office d'un drapeau claquant au vent ${ }^{41}$.

Dans Harper au Congo, le caricaturiste André-Philippe Côté illustre Stephen Harper en visite diplomatique en République du Congo (fig. 8). Dans le registre supérieur de l'œuvre, Harper trottine sereinement, tenant sous son bras gauche ce qui pourrait être une mallette de travail. Derrière lui, un lion s'ap- prête à bondir, toutes griffes dehors. Dans le registre inférieur, le premier ministre pivote sur lui-même sans arrêter son pas; il révèle son arme secrète en affichant un sourire en coin, déjà satisfait d'une victoire assurée. Le fauve sursaute effectivement vers l'arrière en poussant un cri de surprise, apeuré devant le portrait de la reine. Pour une fois, le comportement du premier ministre n'est pas vain et le portrait s'avère efficace. Le lion fuit devant la représentation royale. Ce n'est cependant qu'un animal qui mord à l'hameçon, et non un humain. Dans cette caricature, le premier ministre se trouve sur le continent des fétiches, l'Afrique. C'est sur ce territoire que, au XVII ${ }^{e}$ siècle, les explorateurs portugais ont dénoncé les objets cultuels des Africains qu'ils appelèrent des fétiches, comme le rappelle Bruno Latour. Les explorateurs contestaient les pouvoirs magiques de ces objets en argumentant qu'ils étaient conçus par la main de l'homme (dans leur dénonciation, les Portugais oubliaient leurs propres fétiches, telles que les reliques saintes qu'ils portaient autour du cou) ${ }^{42}$. Dans les caricatures québécoises, Harper est critiqué, tout comme les Africains l'ont été par les Portugais. Son fétiche sert peut-être à effrayer les animaux, mais le portrait de la reine ne réussit pas à régler les problèmes politiques, religieux ou environnementaux. Le trou de la couche d'ozone n'a pas été colmaté. Le Canada n'a toujours pas l'autorité sur le pôle Nord. Le litige entourant la présence du crucifix à l'Assemblée nationale n'est pas résolu. Manipulé par le premier ministre, le portrait de la reine demeure un fétiche caduc.
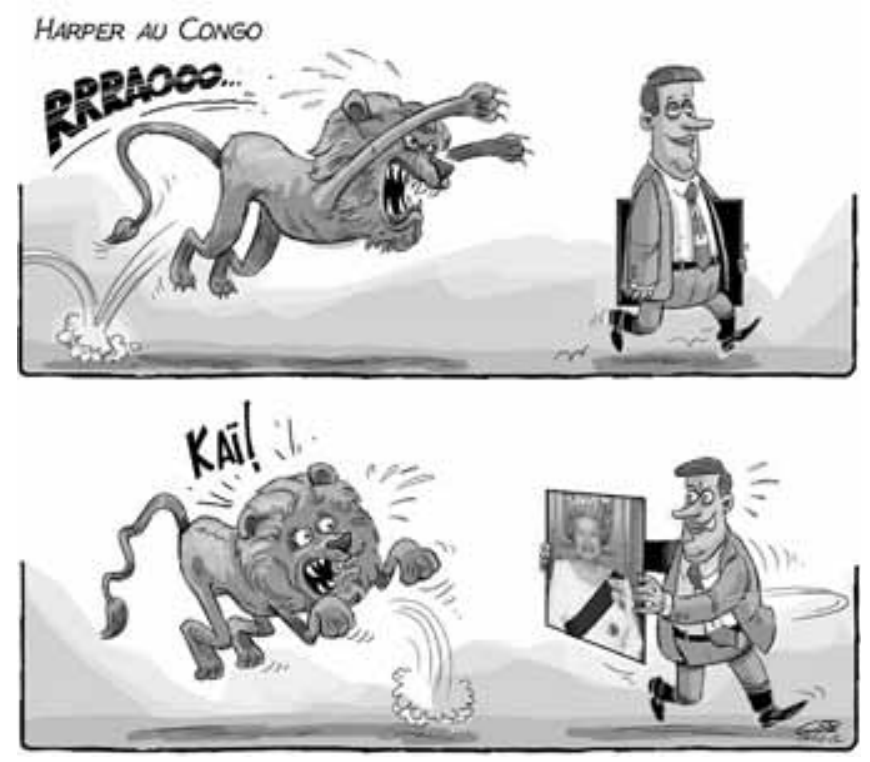

Figure 8. André-Philippe Côté, Harper au Congo, Le Soleil, I4 octobre 2012, avec l'amabilité de l'artiste. 


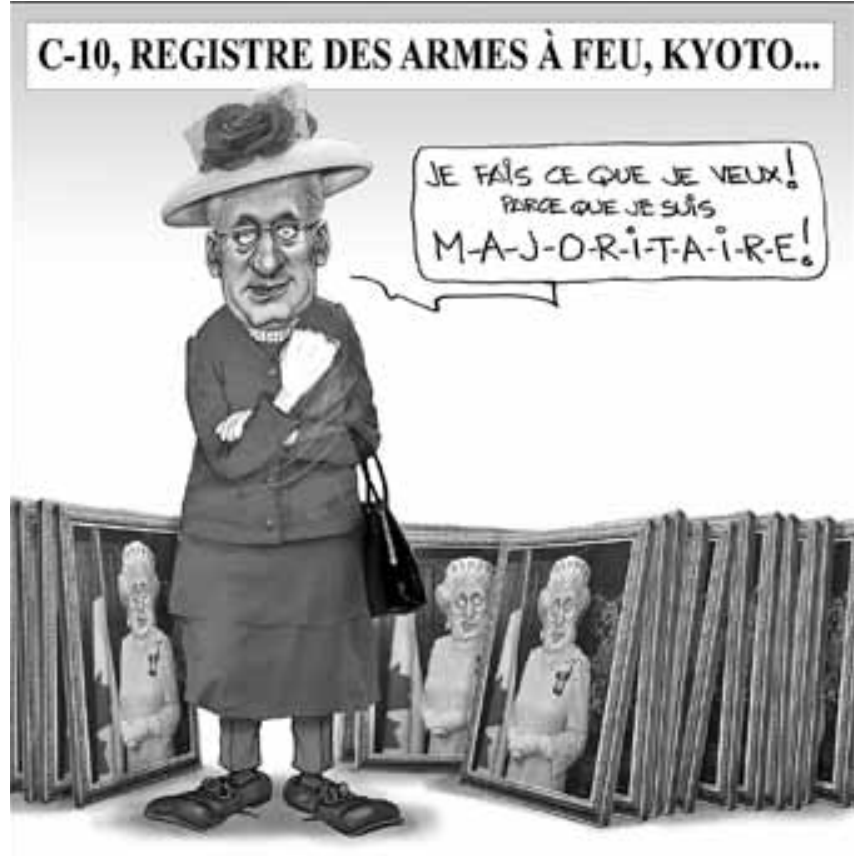

Figure 9. Serge Chapleau, C-I0, registre des armes à feu, Kyoto, La Presse, 15 décembre 2011.

\section{La force économisée du portrait de la reine}

En plus d'être investi du double pouvoir de la représentation la substitution et la légitimation - le portrait de la reine constitue aussi le pouvoir suprême au Canada. Selon Marin, le pouvoir n'est rien d'autre qu'une " mise en réserve de la force dans les signes 43 ", une démonstration d'une force économisée. Le pouvoir implique une contrainte, une loi, qui doit être respectée sous peine d'avoir recours à cette force contenue qui passerait, une fois en action, par un affrontement : " La force n'est force que par destruction, annihilation de toute autre force $[\ldots]^{44}$ ». En s'appropriant le portrait de la reine comme instituteur de son propre pouvoir politique, le premier ministre Stephen Harper (dont le titre incarne déjà une certaine force législative) s'arroge symboliquement la force économisée de la souveraine. Questionner l'autorité du premier ministre revient à questionner le pouvoir de la reine et se risquer à être confronté à la force réunie de la souveraine et du premier ministre. D'ailleurs, l'utilisation du portrait de la reine, qui représente la plus haute instance du pays, est soumise à des restrictions au Canada. Il est interdit de représenter les membres de la famille royale sur des autocollants, des vêtements, de la lingerie, des emballages, des boites, des étiquettes ou sur n'importe quels articles qui auraient des

\section{HARPER VEUT RAVOIR LE PORTRAIT DE LA REINE PRÊTÉ AU MINISTÈRE DES AFFAIRES ÉTRANGÈRES}

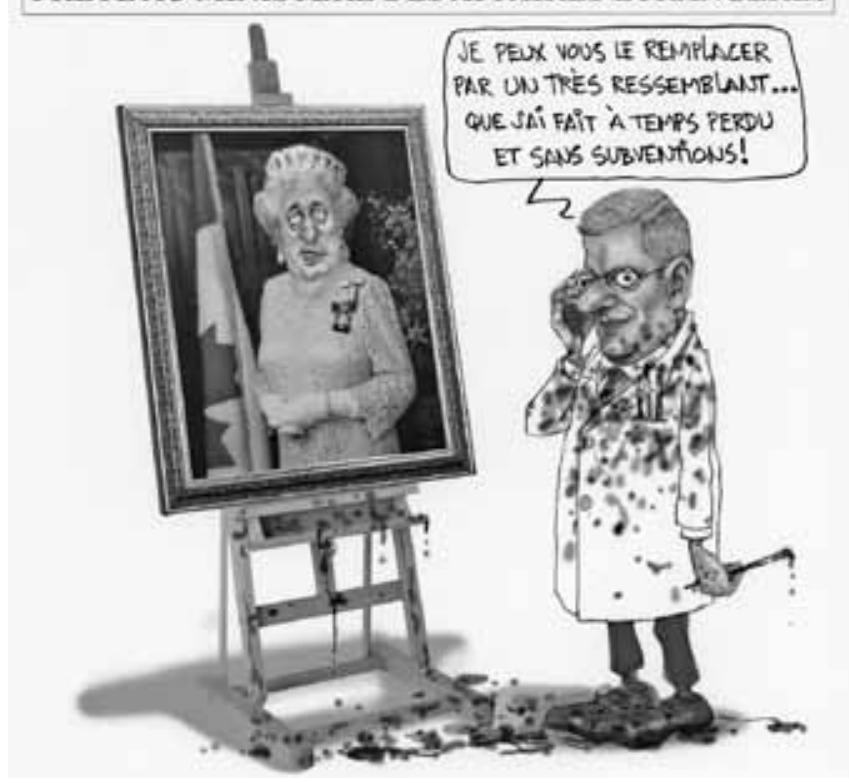

Figure 10. Serge Chapleau, Harper veut ravoir le portrait de la reine prêté au ministère des Affaires étrangères, La Presse, 29 novembre 2011, Musée McCord, M2012.52.50. fins publicitaires ${ }^{45}$. Ces restrictions confèrent au portrait royal un pouvoir : faut-il craindre des représailles de la part de l'État si des images représentant la reine sont entachées par une utilisation à mauvais escient? Heureusement pour les caricaturistes, la législation concernant l'utilisation des portraits royaux ne fait aucune mention de leurs reprises dans les satires graphiques.

Le personnage du premier ministre arrive parfois à donner vie au portrait royal et à en faire une véritable menace dans les caricatures québécoises. Cette tactique, qui va à l'opposé de la présentation du portrait de la reine comme un objet vide de pouvoir, permet tout autant de critiquer les pouvoirs attribués au portrait de la reine par leur mise en valeur dans les établissements gouvernementaux. Dans ces caricatures, le ridicule du personnage de Stephen Harper fait place à l'autoritarisme du premier ministre. L'humanisation du portrait de la reine se produit sous la plume de Chapleau, lorsque ce dernier met en scène le personnage de Stephen Harper qui fusionne avec la représentation de la reine. Dans une caricature datée du 15 décembre 2011 et intitulée C-10, registre des armes à feu, Kyoto, le premier ministre est travesti en reine (fig. 9). Mais l'amalgame entre Harper et Élisabeth II ne se produit pas tant dans le déguisement d'Harper que dans la présence, aux pieds de ce dernier, d'exemplaires de la photographie qui a remplacé les deux 


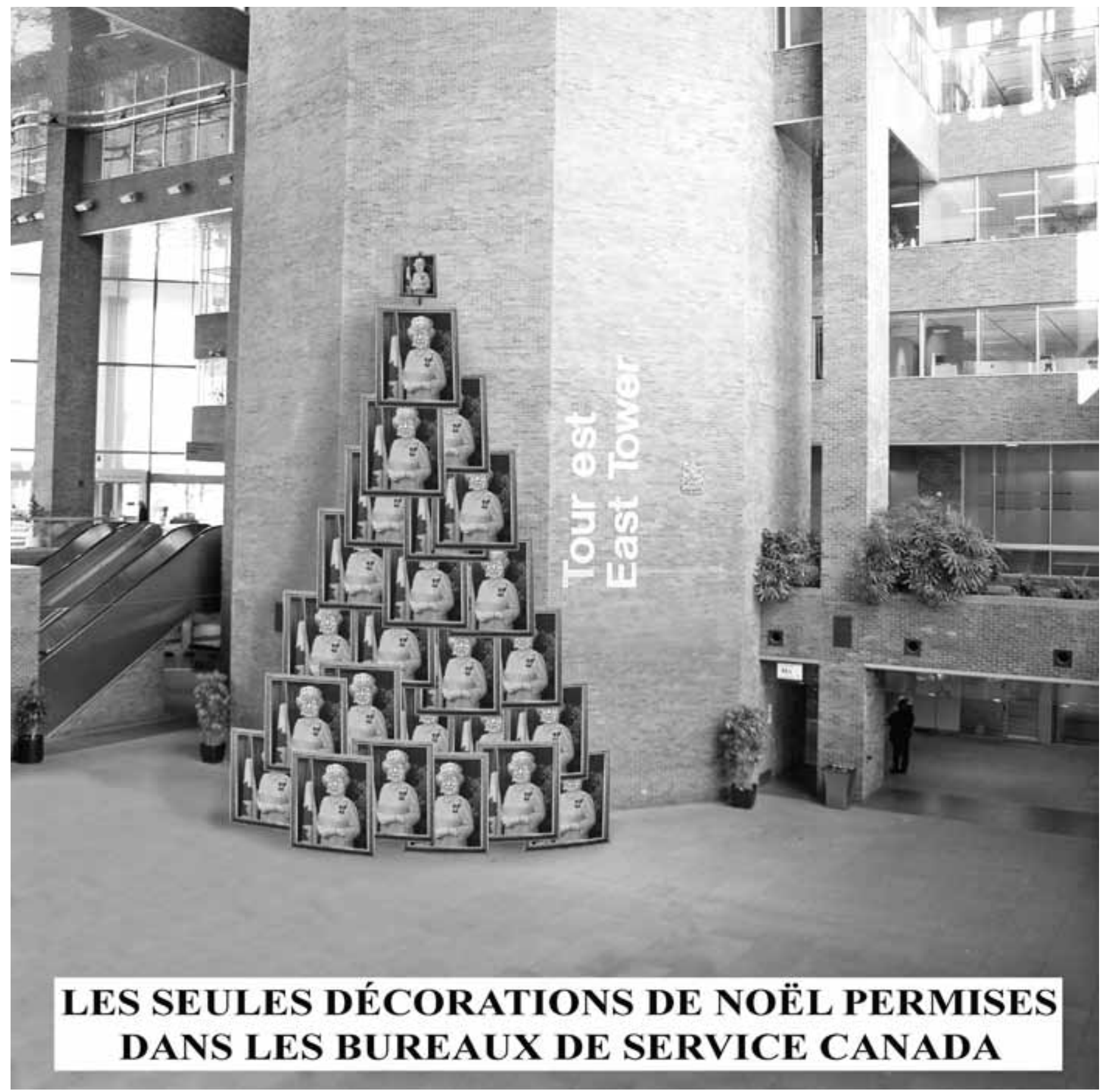

Figure II. Serge Chapleau, Les seules décorations de Noël permises dans les bureaux de Service Canada, La Presse, 3 décembre 201 I. 
œuvres de Pellan dans le hall d'entrée du MAECD. Les portraits sont reconnaissables grâce à certains éléments iconographiques et compositionnels : le fond bleuté, la présence du drapeau canadien et d'un bouquet de fleurs en arrière-plan, la pose de la souveraine, sa robe blanche, ses insignes et sa couronne. Toutefois, la reine n'exhibe pas son vrai visage dans la caricature de Chapleau, elle a été caricaturée...

La source de cette caricature se trouve dans un autre dessin du même caricaturiste, produit près de deux semaines avant celui-ci et intitulé Harper veut ravoir le portrait de la reine prêté au ministère des Affaires étrangères, qui révèle l'instigateur de la défiguration (fig. 10). Le personnage du premier ministre est représenté devant un chevalet sur lequel est placé le portrait de la reine retouché. Tenant un pinceau à la main et vêtu d'un sarrau blanc maculé de taches de peinture bleue, verte et mauve, Harper propose sa création au ministère des Affaires étrangères afin de ravoir son portrait de la reine : " Je peux vous le remplacer par un très ressemblant... que j'ai fait à temps perdu et sans subventions! 46 \#. Les propos du premier ministre relèguent la peinture à un simple divertissement qui ne demande aucun soutien financier, justifiant d'un même élan les coupes budgétaires du gouvernement conservateur dans le domaine de la culture. Résultat : l'œuvre produite par Harper est maladroite. Chapleau ridiculise le manque d'adresse du premier ministre barbouillé de peinture, qui n'arrive pas à représenter correctement le visage de la reine, à moins que la déformation soit volontaire... Dans sa peinture, Harper a modifié le portrait royal pour lui donner ses propres traits. Les yeux tombants et cernés de la souveraine, son long nez volumineux, sa bouche tordue et son double menton sont presque identiques aux traits du premier ministre dans la caricature $C-10$, registre des armes à feu, Kyoto. Deux représentations de pouvoir - une reine et un premier ministre - sont donc combinées dans l'œuvre peinte par Harper, qui devient un créateur réussissant, cette fois-ci, à insuffler une vie à la représentation de la reine ${ }^{47}$. D'ailleurs, ce même portrait prend la parole dans une autre œuvre de Chapleau datée du 29 juillet 2014; il devient ainsi à la fois objet et sujet. Dans ces trois caricatures, le portrait de la reine devient donc une condensation du portrait de la reine et de celui du premier ministre. La fusion iconique se substitue ainsi au portrait de la reine, lui-même un remplacement de la souveraine.

Cette condensation revient dans d'autres caricatures de Chapleau, notamment dans un dessin datant du 3 décembre 2011 intitulé Les seules décorations de Noël permises dans les bureaux de service Canada (fig. 11). Dans cette caricature, la nouvelle icône se multiplie pour prendre la forme pyramidale d'un sapin de Noël. La satire renvoie à une controverse à propos de l'exposition de symboles chrétiens dans les édifices fédéraux. Le 9 novembre 2011, le directeur général de Service Canada au Québec a interdit à ses employés de décorer leurs aires de travail

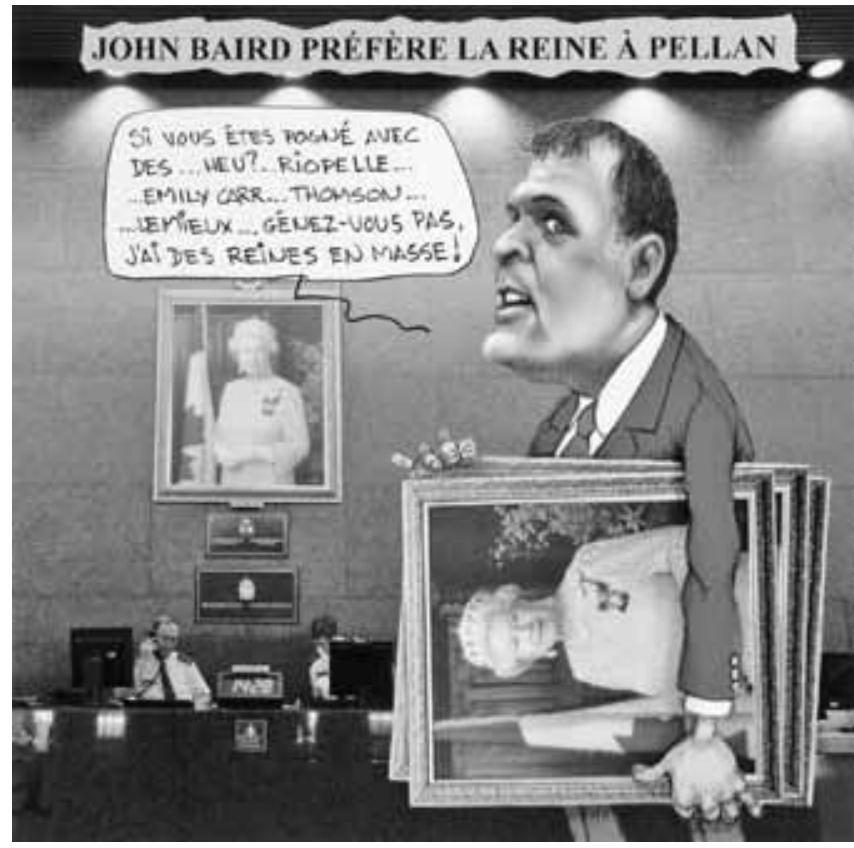

Figure 12. Serge Chapleau, John Baird préfère la reine à Pellan, La Presse, 28 juillet 20II, Musée McCord M20II.100.184.

afin de ne pas offenser les citoyens non chrétiens ${ }^{48}$. Dans le dessin de Chapleau, le portrait royal est à nouveau proposé pour résoudre un débat sur la laïcisation dans un contexte fédéral et non provincial cette fois-ci. En revanche, contrairement à la caricature de Garnotte dans laquelle Harper propose le portrait de la reine à titre de substitut au crucifix de l'Assemblée nationale du Québec, le changement est déjà effectué, comme si le portrait s'était lui-même multiplié et agencé en forme de sapin. Dans les deux cas, le portrait est présenté en tant que substitut à des symboles déjà en place. En ce sens, les caricatures de Chapleau et de Garnotte rappellent le contexte qui a fait naittre le portrait de la reine en tant que motif récurrent dans l'intercaricaturalité québécoise, et cela, même si les deux œuvres de Pellan ne sont pas présentes ${ }^{49}$. En réitérant le comportement du remplacement, ces caricatures dénoncent le pouvoir (en tant que force plus ou moins contenue) qu'a le gouvernement conservateur de changer l'iconographie canadienne en plus de rappeler le rapport de substitution qui subsiste au sein même de la représentation.

\section{Une guerre d'images}

Les pouvoirs des portraits de la reine Élisabeth II qui se trouvent dans les édifices gouvernementaux sont donc différemment exploités dans les caricatures québécoises selon le message à 
véhiculer. S'il est question de ridiculiser l'intérêt monarchique du premier ministre, la représentation royale perd ses pouvoirs législatifs. Par contre, le portrait de la reine conserve son pouvoir de substitution lorsque la réorganisation des images par le gouvernement conservateur est en jeu. Le portrait remplace des symboles religieux comme le crucifix de l'Assemblée nationale du Québec et le sapin de Noël. Il menace même de prendre la place d'autres ouvres d'art dans un montage de Serge Chapleau intitulé John Baird préfere la reine à Pellan (fig. 12). L’ancien ministre des Affaires étrangères y est représenté avec une pile de photographies de la reine sous le bras, annonçant au lecteur : " Si vous êtes pogné avec des... heu? ... Riopelle... ... Emily Carr... Thomson... ... Lemieux... gênez-vous pas, j’ai des reines en masse! ». Jean-Paul Riopelle (1923-2002), Emily Carr (1871-1945), Tom Thomson (1877-1917) et Jean-Paul Lemieux (1904-1990) sont ici mentionnés au même titre qu'Alfred Pellan, soit en tant que créateurs d'œuvres d'art symbolisant le Canada et dont le sort est incertain. Les amoncèlements du portrait de la reine dans $C-10$, registre des armes à feu, Kyoto, dans Les seules décorations de Noël permises dans les bureaux de service Canada et dans John Baird préfere la reine à Pellan deviennent des armées de clones prêtes à remplacer d'autres symboles politiques et religieux. Ces nouveaux emblèmes laissent planer une menace : qui seront les prochaines victimes?

Les caricatures déploient deux tactiques devant cette menace. D'une part, elles ridiculisent le personnage du premier ministre en le représentant comme ayant un comportement fétichiste dysfonctionnel. D'autre part, elles font du motif du portrait royal une arme investie d'un redoutable pouvoir. En effet, loin d'être seulement un objet vide de sens, la représentation du portrait de la reine, de par son autonomisation et sa récurrence dans l'intercaricaturalité québécoise, acquiert le pouvoir de consolider à tout instant et dans toutes les situations l'intérêt politique du gouvernement conservateur envers une monarchie britannique réactivée, mais inanimée. De plus, si la présence du portrait de la reine s'est estompée dans les caricatures québécoises depuis quelques mois, le motif peut ressurgir à tout instant pour dénoncer d'autres substitutions iconiques opérées par le gouvernement et déclencher ainsi une nouvelle guerre d'images.

\section{Notes}

1 La plupart des informations concernant la substitution proviennent d'une demande d'accès à l'information faite à l'attention du ministère des Affaires étrangères, Commerce et Développement Canada (Demande d'accès à l'information numéro A-2012-00445, juillet 2012), ci-après AECDC. Les noms du ministre et du premier ministre ne sont pas explicitement évoqués dans ces documents et certaines informations sont caviardées, ce qui laisse place à des suppositions. La demande aurait été formulée par l'assistant exécutif de John Baird, Rick Roth, dans un appel téléphonique avec Robert Cousin, directeur des services de gestion des locaux, d'audiovisuels, et des conférences. Rien ne permet d'affirmer que l'idée proviendrait directement de Stephen Harper. Par contre, le consentement du Cabinet du premier ministre était nécessaire à l'opération, puisque c'est à lui qu'appartient le portrait de la reine.

2 Jennifer Ditchburn, "John Baird préfere la reine à Alfred Pellan ", La Presse, 26 juillet 2011, http://www.lapresse.ca/actualites/politique/politique-canadienne/201107/26/01-4421201-john-bairdprefere-la-reine-a-alfred-pellan.php.

3 Ibid. Les articles de Ditchburn, qui écrit pour La Presse canadienne, sont repris par les grands journaux locaux un peu partout au Canada.

4 Caricaturistes dont la production a été dépouillée entre janvier 2010 et décembre 2014 : Garnotte (Michel Garneau) dans Le Devoir; Aislin (Terry Mosher) et Pascal Elie dans The Gazette; Marc Beaudet dans Le Journal de Montréal; Ygreck (Yannick Lemay) dans Le Journal de Québec; Jean Isabelle dans Le Nouvelliste; Serge Chapleau dans La Presse; André-Philippe Côté dans Le Soleil; Hervé Philippe dans La Tribune; Yvon Roy dans Voir; et Fleg (Christian Daigle) sur le site internet Yahoo! Actualités Québec. Une seule caricature représentant le portrait de la reine a été publiée entre le $1^{\text {er }}$ décembre 2010 et le 25 juillet 2011, il s'agit d'une caricature d'Aislin datée du 5 avril 2011 dont il sera question plus loin dans cet article. À titre de comparaison, seulement cinq caricatures ontariennes produites dans la même période mettent en scène le portrait litigieux dans The Toronto Sun, The Toronto Star et The Globe and Mail, alors que Le Devoir, La Presse et Le Journal de Montréal en publient trente-six à eux seuls. Les caricaturistes ontariens représentent plutôt la reine comme un personnage animé alors que sa réification sous forme de portrait est un trope particulier à la production caricaturale québécoise. Cinq caricatures québécoises ont représenté la reine comme un personnage animé comparativement aux soixante-trois caricatures de son portrait.

5 Malorie Beauchemin, "Portrait de la reine dans les ambassades : "un vestige de colonialisme" ", La Presse, 7 septembre 2011, http:// www.lapresse.ca/actualites/politique/politique-canadienne/ 201109/07/01-4432411-portrait-de-la-reine-dans-les-ambassades-un-vestige-de-colonialisme.php et Jocelyne Richer, " Le PQ veut rapatrier les deux Pellan retirés à Ottawa ", La Presse, 27 juillet 2011, http://www.cyberpresse.ca/actualites/quebeccanada/national/201107/27/01-4421461-le-pq-veut-rapatrierles-deux-pellan-retires-a-ottawa.php?utm_categorieinterne=traffic drivers\&utm_contenuinterne=cyberpresse_vous_suggere_ 4421201_article_POS1.

6 Cet article s'arrime à trois façons d'aborder la caricature. D'abord, par l'analyse d'un motif de la même manière que le fait Ségolène Le Men dans son article " Gravures, caricatures et images cachées : la genèse du signe du roi en Poire ", Genesis, manuscrits, recherche, invention, $\mathrm{n}^{\circ} 24,2004$, p. 43-69, soit en faisant la genèse de la 
poire dessinée par le caricaturiste et éditeur Charles Philipon en 1831 lors d'un procès pour libelle contre le roi. Le croquis, une caricature en quatre temps montrant la transformation du roi Louis-Philippe en poire qui n'a guère aidé le caricaturiste dans sa cause, a été largement diffusé et s'est transformé à travers le temps. C'est également en ce sens que j'emprunte le néologisme intercaricaturalité au Groupe de recherche sur la caricature (2005-2007) dirigé par Todd Porterfield, professeur au Département d'histoire de l'art et d'études cinématographiques de l'Université de Montréal. Considérer le portrait de la reine comme un motif intercaricatural permet de se dégager du propos des caricaturistes et des lignes de parti des différents journaux en jeu, non pas parce que cette méthodologie n'est pas pertinente, mais bien pour soulever un phénomène iconique plus large qui est la multiplication du motif du portrait peu importe l'auteur et le lieu de publication. Finalement, cet article s'ancre aux recherches de Martha Bantha en étudiant les comportements d'un personnage, ici le premier ministre Stephen Harper, comme éléments révélateurs d'une certaine anxiété sociale. Martha Banta, Barbaric Intercourse : Caricature and the Culture of Conduct, 1841-1936, Chicago et Londres, The University of Chicago Press, 2002.

7 Coupure pure est un jeu de mots utilisé par Jacques Derrida pour nuancer la distinction kantienne qui divise l'ergon (l'œuvre) et le parergon (ce qui est à l'extérieur de l'œuvre). Jacques Derrida, "Parergon ", La vérité en peinture, Paris, Flammarion, Coll. Champs d'essais, 1978, p. 66-71. Il ne peut y avoir une coupure pure entre le motif caricatural du portrait et son contexte d'émergence, de la même manière que le contexte de la substitution ne peut être totalement dissocié de la photographie de la reine et des œuvres de Pellan.

8 Les coupes en culture n'indiquent en rien un désintérêt de la chose artistique chez les Conservateurs comme l'ont démontré Simon Jodoin, Steve Proulx, Anabelle Moreau et Pedro Despouy, dans "Art-peur ", Voir, 6 février 2013, p. 8-9. De nombreux monuments ont vu le jour, ou sont en cours de réalisation, en collaboration avec différents organismes et fondations (des monuments dédiés aux animaux de la guerre, à la guerre de 1812, aux victimes du communisme, aux soldats), sans compter la création de bandes dessinées qui racontent la guerre de 1812, la commande d'un vitrail à l'effigie des reines Élisabeth II et Victoria ainsi que d'une acrylique sur toile de la reine Élisabeth II dont il sera question dans cet article. Hormis l'affaire des Pellan, d'autres substitutions iconographiques ont eu lieu dans des édifices gouvernementaux, notamment à Rideau Hall, ou encore sur la monnaie canadienne. L'architecture du Musée canadien des civilisations conçue par Douglas Cardinal a, pour sa part, eu droit à un changement de nom et de fonction.

9 Par portrait, j'entends ici - très largement il est vrai - le genre pictural. Ce qui ne veut pas dire qu'il n'y a pas d'autres portraits de la reine au Canada dans le sens entendu par Ernst Kantorowicz, Louis Marin, Peter Burke ou encore Gérard Sabatier, c'est-à-dire le portrait royal qui passe globalement par l'histoire du roi, par ses représentants et par ses institutions. Ernst Kantorowicz, Les deux corps du roi. Essai sur la théologie politique au Moyen Age, Paris, Gallimard, 1989; Louis Marin, Le portrait du roi, Paris, les éditions de Minuit, 1981; Peter Burke, Louis XIV. Les stratégies de la gloire, Paris, Seuil, 1995; Gérard Sabatier, "La gloire du roi. Iconographie de Louis XIV de 1661 à 1672 ", Histoire, économie et société, vol. $19, n^{\circ} 4,2000$, p. $527-560$.

10 Le gouverneur général du Canada, "Rapport annuel 2011-2012 », Gouverneur général du Canada, 2011-2012, p. 4, http://www. gg.ca/PDF/AR11-12FR_web_final.PDF.

11 C'est ce que nous apprend Jocelyne Richer dans son article « Le PQ veut rapatrier les deux Pellan retirés à Ottawa ", op. cit.

12 La légation du Canada au Brésil, qui ouvre ses portes en 1941, deviendra une ambassade en 1943. Jean Désy organise une exposition collective regroupant des œuvres d'artistes canadiens qui circule à Rio de Janeiro et à Sao Paulo à la fin de l'année 1944 et au début de l'année suivante. Canada Ouest et Canada Est en font partie et reçoivent une excellente critique. Voir le recueil des articles sur l'exposition Canadian Art in Brazil : Press Review. Art canadien au Brésil : revue de la presse, s. l., s.n., 1945.

13 Selon l'ex-ambassadeur Thomas Delworth, Pellan aurait donné Canada Est et Canada Ouest à Désy après que celui-ci lui ait permis d'étudier à l'école des beaux-arts de Rio de Janeiro. AECDC, op. cit., p. 55. Aucune mention de ce voyage d'études n'a été relevée dans les monographies de l'artiste qui annoncent plutôt que Désy a commandé les deux œuvres à Pellan. Guy Robert, Pellan, sa vie et son ceuvre / His Life and His Art, Montréal, éditions du centre de psychologie et de pédagogie, 1963, p. 39; Germain Lefebvre, Pellan, sa vie, son art, son temps, La Prairie, Marcel Broquet, 1986 , p. 115-116; Michel Martin et Sandra Grant Marchand (dir.), Alfred Pellan, Catalogue de l'exposition présentée au Musée d'art contemporain de Montréal du 11 juin au 26 septembre 1993 et au Musée du Québec du 13 octobre 1993 au 30 janvier 1994, Montréal et Québec, Musée d'art contemporain de Montréal et Musée du Québec, 1993, p. 76; Jacques Lamarche, Alfred Pellan, Montréal, Lidec, 1997, p. 37. L'ouvrage de Maurice Gagnon, Pellan, Montréal, L'Arbre, 1943, fait mention des œuvres de Pellan sans aucune information sur leur contexte de création.

14 Marie Carani, "De l'euphorie au silence ", dans Martin et Marchand (dirs.), Alfred Pellan, op. cit., p. 221-244.

15 Marie Vastel, "Retour discret des toiles de Pellan aux Affaires étrangères ", Le Devoir, 29 juillet 2014, http://www.ledevoir.com/ politique/canada/414635/retour-discret-des-toiles-de-pellanaux-affaires-etrangeres.

16 Ditchburn, op. cit.

17 AECDC, op. cit., p. 9

18 Michael Bedford a photographié la souveraine à quatre reprises au cours de sa carrière. Joel Bedford, "Queen Elizabeth 60th Jubilee Portrait Session (Behind the Scenes) ", Jbedfordphoto, 
3 juin 2012, http://jbedfordphoto.wordpress.com/2012/06/03/ queen60th/\#jp-carousel-96. Le portrait de 2002 a été dévoilé en juin 2003 dans le foyer du Sénat. Il aurait pris place dans le Musée du portrait du Canada si le projet muséal n'avait pas été avorté en 2006 lors de l'arrivée du Parti conservateur au pouvoir. Sénat du Canada, Rapport annuel du Sénat du Canada 2003-2004, Sénat du Canada, 2004, p. 39.

19 Alec Castonguay, "La face cachée du plan Harper ", L'Actualité, 24 février 2012, http://www.lactualite.com/actualites/politique/ la-face-cachee-du-plan-harper/.

20 Les responsables du remplacement des œuvres de Pellan, Daniel Sharp de la collection des arts visuels et Robert Cousin, ont songé à réinstaller les œuvres de Pellan ailleurs dans l'édifice Pearson. Il semble que leurs projets aient été vains. AECDC, op. cit., p. 6-9 et 21 .

21 Rien n'est mentionné sur le montant octroyé pour le retour du portrait au Parlement ni sur le coût du tirage d'une nouvelle photographie. À titre d'exemple, le transport des Pellan vers un entrepôt, le transfert du portrait du Parlement au ministère (fait par Art Zone Fine Art Service) ainsi que l'accrochage du portrait de la reine et ses deux plaques (fait par SNC-Lavalin) ont été évalués à 4655 \$. AECDC, op. cit., p. 29 et 42.

22 Il est difficile d'employer ce mot sans évoquer Walter Benjamin pour qui la valeur ajoutée du portrait de la reine ne serait pas nécessairement une anomalie. En effet, selon Benjamin, le portrait photographique conserve un "dernier éclat d'aura ". Walter Benjamin, L'ouvre d'art à l'époque de sa reproduction technique, Paris, Allia, 2004, p. 32. Or, le Cabinet du premier ministre semble avoir une préférence particulière pour la photographie « originale » par rapport aux autres tirages, laissant aux personnes travaillant au ministère des Affaires étrangères, Commerce et Développement Canada le soin de se procurer une réplique. Il y a peu d'information sur le cadre de la photographie d'" origine " qui pourrait expliquer la prédilection du Cabinet.

23 La substitution de l'œuvre de Morrisseau par celle de Richards n'a pas été produite dans les mêmes circonstances que le remplacement des Pellan. D'abord, l'évènement était prévu depuis longtemps. Androgyny avait été prêtée à Rideau Hall par la collection nationale d'art indien des Affaires autochtones et Développement du Nord Canada (AADNC) et le prêt était échu depuis un an lors de l'arrivée du portrait. Aussi, le remplacement ne s'est pas produit en catimini. Le portrait de la reine a eu droit à deux inaugurations. Un dévoilement en grande pompe par le sujet de l'œuvre, la reine Élisabeth II, a eu lieu au palais de Buckingham le 6 juin 2012, soit pendant la semaine qui célébrait son jubilé de diamant. L'œuvre a été inaugurée une seconde fois le 28 juin 2012, dans la salle de bal de Rideau Hall, devant les journalistes. Néanmoins, le choix de mettre un portrait de la reine à cet endroit s'inscrit dans la lignée des changements iconographiques qui ont eu lieu au MAECD et dans les ambassades canadiennes.
24 Ce fut le cas de l'assermentation qui a eu lieu lors du remaniement ministériel en juillet 2013. Lorsqu'il y a de petits changements au sein du Cabinet, la cérémonie peut être faite dans d'autres pièces de Rideau Hall comme le petit salon.

25 L'image de la reine est tout de même restée présente au Canada, notamment sur les billets de vingt dollars et sur les pièces de monnaie. De plus, ces moments ont été beaucoup plus complexes qu'une simple distanciation vis-à-vis de la Grande-Bretagne. Les provinces canadiennes, les peuples autochtones, le gouvernement fédéral, la monarchie et le Parlement britannique ainsi que d'autres instances à l'international (notamment les États-Unis) ont participé à faire de ces moments charnières des nœuds politiques, culturels et économiques. Parmi les récents écrits qui soulignent la complexité de ces évènements clé de l'histoire canadienne se trouve l'ouvrage de Ian McKay et Jamie Swift, Warrior Nation : Rebranding Canada in an Age of Anxiety, Toronto, Between the Lines, 2012, dans lequel les auteurs démystifient la politique internationale du premier ministre Lester Pearson tout en soulignant son attachement pour la monarchie britannique. De son côté, Frédéric Bastien dans $L a$ Bataille de Londres, Montréal, Boréal, 2013, revisite le rapatriement de la Constitution qui ne s'est pas faite sans heurts, tant à Ottawa qu'à Westminster. Aussi, Ian Peach dans son article «The Power of a Single Feather : Meech Lake, Indigenous Resistance and the Evolution of Indigenous Politics in Canada ", Review of Constitutional Studies/Revue d'études constitutionnelles, vol. 16, $\mathrm{n}^{\circ} 1$, 2011, p. 1-29, insiste sur l'importance de la résistance autochtone lors de l'accord du Lac Meech, force souvent oubliée en faveur des revendications québécoises. Enfin, pour un aperçu global sur les rapports tumultueux entre les provinces canadiennes, le gouvernement fédéral et l'Angleterre, il est toujours bon de se référer aux textes de Peter Waite, «Un défit continental 1840-1900 », Ramsay Cook, "Triomphe et revers du matérialisme 1900-1945 " et Desmond Morton, "Crises d'abondance 1945-1988 ", dans Histoire générale du Canada, Craig Brown (dir.), Montréal, Boréal, 1990, 333-678, qui demeurent d'actualité.

26 En août 2011, le ministre de la Défense nationale Peter MacKay remet en fonction les désignations "Corps d'aviation royal canadien » et " Marine royale canadienne " qui avaient été réunies (avec l'Armée canadienne) sous le nom des Forces canadiennes le $1^{\text {er }}$ février 1968.

27 Il s'agit de la théorie exposée par Ian McKay, Jamie Swift et Yves Frenette. Voir McKay et Swift, op. cit. et Yves Frenette, «Conscripting Canada's Past : The Harper Government and the Politics of Memory ", Canadian Journal of History, vol. 49, $\mathrm{n}^{\circ} 1$, printemps 2014, p. 49-65.

28 Marin, op. cit., p. 10-12.

29 Antonio Pinelli, Christine Tauber, et al., « Le portrait du roi : entre art, histoire, anthropologie et sémiologie ", débat, Perspective, vol. 1, 2012, p. 15-16.

30 Burke, op. cit., p. 19. 
31 Garnotte, Le Devoir, 3 octobre 2011, 20 décembre 2011, 18 janvier 2012, 20 janvier 2012; Élie, The Gazette, 25 juillet 2013; Côté, Le Soleil, 20 novembre 2011; Philippe, La Tribune, 11 décembre 2012; Isabelle, Le Nouvelliste, 11 juin 2013.

32 Garnotte, Le Devoir, 9 septembre 2011, 9 octobre 2011, 7 mars 2012, 8 juin 2013.

33 Le fétichisme peut faire partie d'une vie amoureuse normale s'il n'est pas l'unique objet sexuel, nuance Sigmund Freud dans Trois essais sur la théorie sexuelle, Paris, Gallimard, Coll. "Folio essais ", 1987, p. 63. Cependant, compte tenu de l'impossibilité d'une véritable union sexuelle entre le premier ministre et la reine, le fétichisme d'Harper devient ici une déviance.

34 Autres caricatures qui montrent le premier ministre avec ces apparats en présence du portrait de la reine : Garnotte, Le Devoir, 28 avril 2011 et 19 août 2011 .

35 Une copie de l'œuvre se trouve à Rideau Hall. Cette caricature d'Aislin rappelle que le personnage de Stephen Harper est luimême un portrait dans les caricatures et que celles-ci sont souvent encadrées afin de les distinguer du texte qui les entoure dans un journal ou sur une page web. La différence entre le portrait de la reine et celui de Stephen Harper ne réside donc pas tant dans la présence ou non d'un cadre, mais dans le comportement des protagonistes à l'intérieur de leur cadre respectif qui les isole l'un de l'autre.

36 Voir Garnotte Le Devoir, 25 septembre 2012 et Côté, Le Soleil, 21 juin 2014.

37 Marin, op. cit., p. 10-11.

38 Kantorowicz, op. cit., p. 54, 143. S'il n'est pas question dans cet article de l'autre corps de la reine, son corps « naturel ", Kantorowicz ouvre très certainement une brèche vers une étude plus panofskienne des deux corps de la souveraine à travers ses représentations, sujet qui mérite sa propre étude.

39 Ce qui ne réduit en rien l'importance de ce rôle dans l'acquisition de la citoyenneté canadienne, par exemple.

40 Gouvernement du Québec, Charte affirmant les valeurs de lä̈cité et de neutralité religieuse de l'État ainsi que d'égalité entre les femmes et les hommes et encadrant les demandes d'accommodement, Québec, Publication du Québec, 2013, p. 2.

41 Des articles sur la menace d'une présence étrangère dans l'Arctique parsèment le journal Le Devoir quelques mois avant la publication de la caricature de Garnotte. Voir entre autres : Alexandre Shields, "Visite de l'ancien premier ministre français - Arctique : la fin du "chacun chez soi" est inévitable, croit Michel Rocard ", Le Devoir, 9 août 2011, http://www.ledevoir.com/politique/canada/328997/ visite-de-l-ancien-premier-ministre-francais-arctique-la-fin-duchacun-chez-soi-est-inevitable-croit-michel-rocard; non signé, «Une motoneige furtive pour l'Arctique », Le Devoir, 22 août 2011, http://www.ledevoir.com/politique/canada/329804/en-brefune-motoneige-furtive-pour-1-arctique; Agence France-Presse, "En bref - Poutine vante les avantages de l'Arctique », Le Devoir,
23 septembre 2011, http://www.ledevoir.com/international/ europe/331963/en-bref-poutine-vante-les-avantages-de-l-arctique.

42 Bruno Latour, Sur le culte moderne des dieux faitiches; suivi de Iconoclash, Paris, La Découverte, 2009, p. 21-23.

43 Louis Marin, op. cit., p. 12.

44 Louis Marin, "Le pouvoir et ses représentations ", dans Politiques de la représentation, Paris, Kimé, 2005, p. 75.

45 Gouvernement du Canada, « Portraits royaux et utilisation d'images ", Couronne canadienne, $1^{\text {er }}$ juin 2012, http://couronnecanadienne.gc.ca/fra/1331810602295/1331812087007\#a3.

46 Chapleau s'insinue dans un débat sur la spécificité des médiums et leur valeur respective. Le personnage de Stephen Harper préfère " ravoir " sa photographie royale plutôt que de garder sa peinture. Il faut dire que pendant l'affaire des Pellan, certains détracteurs - dont Germain Lefebvre, biographe d'Alfred Pellan - ont critiqué le choix qu'a fait l'État de substituer les deux gouaches de Pellan "par une image [reproductible] qui peut être vue ailleurs". Ditchburn, op. cit. Selon le ministre John Baird, le recours au médium de la peinture pour représenter la souveraine avait été envisagé par le gouvernement, mais le coût engendré par une telle production a été jugé trop élevé par rapport au produit fini. AECDC, op. cit., p. 51. La déclaration de John Baird sera contredite un an plus tard, lorsque le gouvernement conservateur commandera une œuvre à Phil Richard pour célébrer le Jubilé de diamant de la reine. Il s'agit de l'acrylique sur toile qui se trouve sur le mur nord de la salle de bal à Rideau Hall.

47 Trois autres caricaturistes présentent Harper comme un artiste : Fleg, Yahoo! Actualités Québec, 28 juillet 2011; Garnotte, Le Devoir, 15 septembre 2012; Côté, Le Soleil, 2 décembre 2013.

48 André Noël, "Pas de décorations de Noël chez Service Canada ", La Presse, 2 décembre 2011, http://www.lapresse.ca/actualites/ politique/politique-canadienne/201112/01/01-4473726-pas-dedecorations-de-noel-chez-service-canada.php. Le gouvernement conservateur rectifiera ensuite le tir en autorisant aux fonctionnaires de l'État de décorer leur lieu de travail : article non signé, "Ottawa dit à Service Canada d'autoriser les décorations de Noël ", Radio-Canada, 2 décembre 2011, http://ici.radio-canada. $\mathrm{ca} /$ nouvelles/societe/2011/12/02/002-decoration-noel-servicescanada.shtml.

49 Sur les soixante-trois caricatures représentant le portrait de la reine, une seule illustre les œuvres d'Alfred Pellan et encore, ces dernières sont montrées de dos (Garnotte, Le Devoir, 28 juillet 2011). L'absence de Canada Est et Canada Ouest dans les caricatures fait écho à leur décrochage des murs du MAECD, mais aussi à leur disparition entre le moment de leur décrochage et leur exposition au Musée national des beaux-arts du Québec. Alors que les porte-paroles du ministre Baird ne savaient dire avec précision où se trouvaient les œuvres en question, des rumeurs concernant la vente des tableaux ont circulé dans les médias. Richer, op. cit.; Claudette Samson, «Le fédéral gardera les tableaux d'Alfred Pellan, 
affirme James Moore ", Le Soleil, 29 juillet 2011, http://www. lapresse.ca/le-soleil/actualites/politique/201107/29/014422107-le-federal-gardera-les-tableaux-dalfred-pellanaffirme-james-moore.php; Jennifer Ditchburn, "Portrait de la reine : Ottawa voulait vendre les tableaux de Pellan ", La Presse, 7 janvier 2012, http://www.lapresse.ca/actualites/politique/ politique-canadienne/201201/07/01-4483703-portrait-de-lareine-ottawa-voulait-vendre-les-tableaux-de-pellan.php. 